\title{
Optimization of positron trapping and acceleration in an electron-beam-driven plasma wakefield accelerator
}

\author{
X. Wang, ${ }^{1}$ P. Muggli, ${ }^{1}$ T. Katsouleas, ${ }^{1}$ C. Joshi, ${ }^{2}$ W. B. Mori, ${ }^{2}$ R. Ischebeck,${ }^{3}$ and M. J. Hogan ${ }^{3}$ \\ ${ }^{1}$ University of Southern California, Los Angeles, California 90089, USA \\ ${ }^{2}$ University of California at Los Angeles, Los Angeles, California 90095, USA \\ ${ }^{3}$ Stanford Linear Accelerator Center, Stanford, California 94025, USA
}

(Received 22 January 2009; published 20 May 2009)

Positron trapping and acceleration in a plasma wake using a four-bunch scheme [X. Wang et al., Phys. Rev. Lett. 101, 124801 (2008)] is numerically investigated through 2D particle-in-cell simulations. This scheme that integrates positron generation, trapping, and acceleration into a single stage is a promising approach for investigating positron acceleration in an electron-beam-driven wake. It consists of a plasma with an embedded thin foil target into which two closely spaced electron beams are shot. The first beam creates a region for accelerating and focusing positrons and the second beam provides positrons to be accelerated. Some of the outstanding issues related to the quality of the accelerated positron beam load are discussed as a function of the beam and plasma parameters. Simulations show that a large number of positrons $\left(10^{7}-10^{8}\right)$ can be trapped when the plasma wake is modestly nonlinear, and the positrongenerating foil target must be immersed into the plasma. Beam loading can reduce the energy spread of the positron beam load. The quality of the positron beam load is not very sensitive to the exact bunch spacing between the drive electron bunch and the positron beam load.

DOI: 10.1103/PhysRevSTAB.12.051303

PACS numbers: 41.75.Ht, 41.75.Lx, 52.40.Mj

\section{INTRODUCTION}

To realize a future plasma-based linear collider, high gradient and high quality acceleration of positron and electron bunches are equally important [1]. Recent experiments and simulations have shown that the highly nonlinear plasma wake driven by a single electron bunch provides a linear focusing force, and a large and uniform acceleration gradient for the trailing electrons [2-4]. Compared to electron acceleration, positron acceleration in a plasma is less studied due to the lack of intense, relativistic positron bunches. One way to accelerate a positron bunch is to accelerate a trailing positron bunch on the plasma wake excited by a drive positron bunch [5]. Some aspects of positron acceleration and focusing in this scheme have been explored and tested experimentally using a single positron bunch [6-9]. However, in the highly nonlinear regime the plasma wake driven by a positron bunch is fundamentally different from that driven by an electron or laser beam. As a result, the acceleration gradient is smaller and the focusing force is nonlinear for the trailing positron bunch, or positron beam load. This is due to the phase mixing of the returning plasma electrons [5]. To obtain a high-energy positron bunch with a high beam quality, the positron bunch can be also accelerated in the wake of an electron beam [10]. Wang et al. [11] have recently proposed a new scheme to test the acceleration of a positron bunch in the plasma wake driven by an electron bunch before suitable electron/positron bunch trains become available from rf accelerators.
This idea can be briefly described as follows (Fig. 1): two closely spaced but relativistic electron bunches are focused on a thin high- $Z$ foil target placed at the entrance of plasma. Positrons are produced through pair creation as

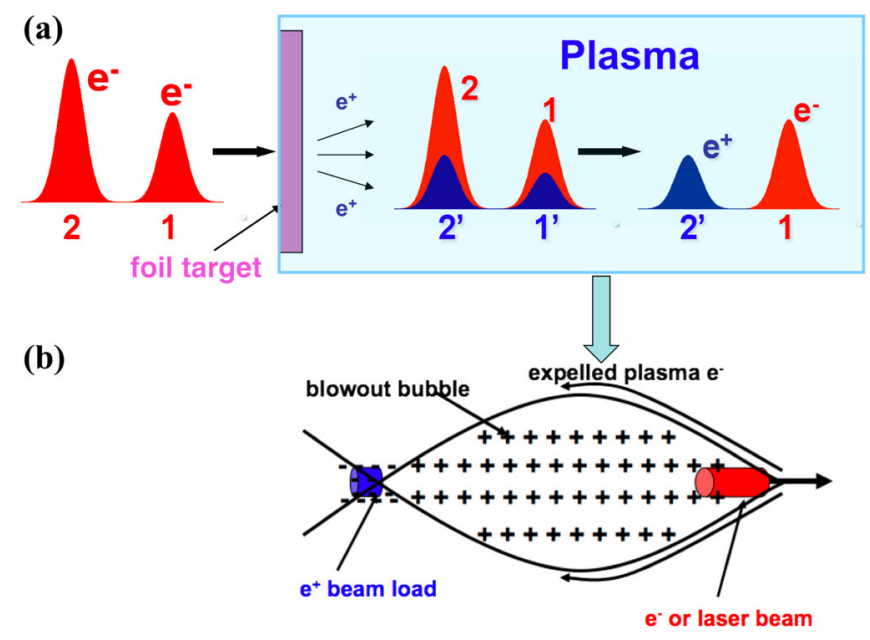

FIG. 1. (Color) Schematic of positron generation, trapping, and acceleration in a plasma, (a) The two incoming electron bunches $(1,2)$ generate in a thin foil target two overlapping (in time and space) positron bunches $\left(1^{\prime}, 2^{\prime}\right)$. After a short distance, the electron driver (1) and the positron beam load $\left(2^{\prime}\right)$ remain in the plasma and the positron driver $\left(1^{\prime}\right)$ and the electron beam load (2) are blown out due to the strong plasma wakefield. (b) The schematic of positron acceleration on the plasma wake driven by an electron or laser beam driver. 
the incoming electron bunches traverse the target. After the target, two positron bunches with a lesser charge than that of the original electron bunches emerge superimposed in space and time onto the electron bunches. The four bunches (electron and positron drive bunches, electron and positron beam loads) propagate downstream into the plasma. For a given plasma density, the distance between the two drive bunches and the two beam loads is adjusted such that the beam loads are placed directly after the blowout bubble created by the electron driver in a region of the plasma wake with excess negative charge. The strong transverse plasma wakefields defocus the electron beam load, as well as the positron drive bunch located in the plasma ion column. Only the electron drive bunch and the positron beam load remain after a short propagation distance into the plasma. In this way, the desirable configuration for accelerating and focusing a tightly focused positron beam load in the wake of an electron beam is established.

This scheme requires the production of two electron bunches closely separated in time $[O(100 \mathrm{fs}-1 \mathrm{ps})]$. A method to produce such bunches, by manipulating a single electron bunch with a correlated energy spread in the dispersive region of the beam line and sending it through a metallic mask, has recently been demonstrated experimentally [12]. A similar method will be used to produce a drive/trailing bunch train for plasma wakefield accelerator (PWFA) experiments at the SLAC National Accelerator Laboratory [13]. The aim is to demonstrate the highgradient acceleration of a witness electron bunch with a narrow (a few percent) energy spread. The two pulse structure expected in the proposed SLAC experiments will have parameters similar to those considered here, which makes this scheme very attractive for investigating positron acceleration in an electron-beam-driven wake.

In this paper, we study in a greater detail than in Ref. [11] the dependence of the positron beam load parameters as a function of the beam and plasma parameters. We first use the code EGS5 to obtain the electron and positron bunch parameters after the foil target. Then we determine the energy threshold for trapping positrons by the plasma wake. Next we show that in general more positrons are trapped in a modestly nonlinear wake than in a highly nonlinear one. We find that the beam loading of the wake can lead to a minimum energy spread for the positron beam load emerging from the plasma, albeit at a small expense to the acceleration gradient. We show that in order to trap a significant number of positrons the foil target must be embedded in the plasma and not be placed in a low-density ramp region preceding the plasma. We find that the number of trapped positrons is not very sensitive to the spacing between the drive and trailing bunches. Finally, we look at this scheme in a plasma field-ionized by the drive electron bunch, as opposed to a preionized plasma that was assumed for the previous study, and find that the continued acceleration of the positron bunch is limited by the beam head erosion of the drive electron bunch.

\section{SIMULATION OF THE POSITRON BUNCH CREATION}

Positron creation in a foil target is a complex physical process and modeled using the advanced Monte-Carlo code electron gamma shower 5 (EGS5) [14]. This code includes Rayleigh scattering, impact and field ionization, bremsstrahlung radiation, pair production, and annihilation, etc. For an incoming bunch containing $1.8 \times 10^{9}$ electrons with an initial energy of $28.5 \mathrm{GeV}$, we choose a $0.5 \mathrm{~mm}$ tantalum foil target $(Z=73$, radiation length $4.1 \mathrm{~mm})$ as a tradeoff between the positron yield and the emittance growth of the incoming electron bunch due to scattering in the target. The EGS5 simulation results of the full phase spaces of the electron bunch and the generated positron bunch show that the electron bunch suffers a small energy loss in the target [Fig. 2(a)], and that the emerging positron bunches, as expected, have an exponential distribution of forward energies [Fig. 2(b)]. The positron yield is about $5 \%$. The angular distributions of the positron bunch in the $x$ and $y$ direction [Figs. 2(d) and 2(f)] follow those of the electron bunch that created it [Figs. 2(c) and 2(e)], with a small fraction of positrons appearing at larger angles. When emerging from the foil target the positron bunches have the same transverse size as the electron bunches. As a result, the geometric emittances (defined as rms transverse size times rms divergence angle) of the electron bunch and the core of the positron bunch are similar.
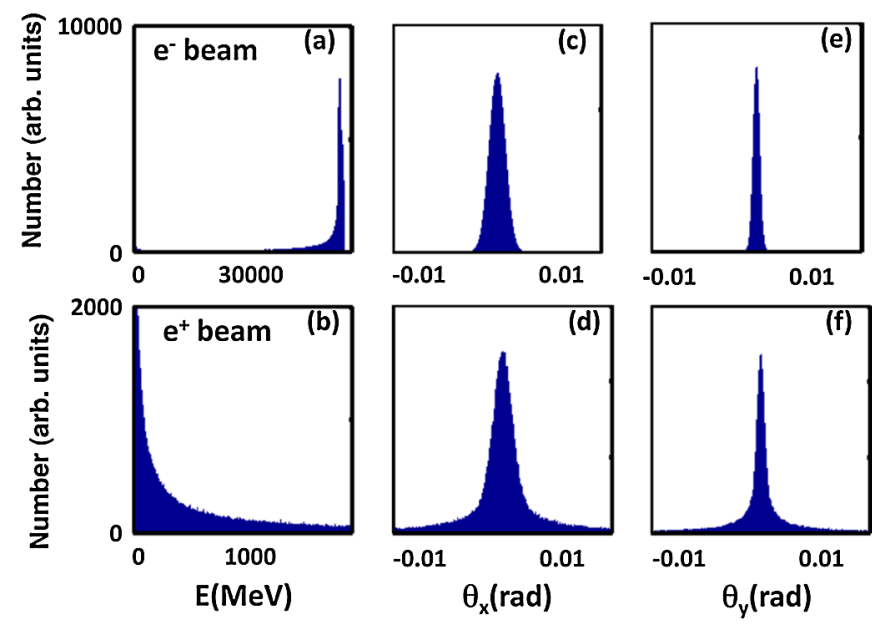

FIG. 2. (Color) EGS5 simulation results of the full phase spaces after a $0.5 \mathrm{~mm}$ tantalum target: energy spectrum of (a) the electron bunch and (b) the produced positron bunch; angular distribution in the (c) $x$ and (e) $y$ direction for the electron bunch, and in the (d) $x$ and (f) $y$ direction for the positron bunch. 
TABLE I. The 2D OSIRIS simulation default parameters. $N_{b}$ is the number of particles in the electron $\left(e^{-}\right)$or positron $\left(e^{+}\right)$bunches. All parameters are given after the foil target.

\begin{tabular}{ll}
\hline \hline Bunch profile $\left(e^{-} / e^{+}\right)$ & Bi-Gaussian \\
$e^{-}$bunches energy $(\mathrm{GeV})$ & 28.5 \\
$e^{+}$beam energy width $(\mathrm{MeV})$ & rms 120 (half Gaussian) \\
$e^{-}$bunches $\varepsilon_{N r} / \varepsilon_{N \theta}(\mathrm{mm} \mathrm{rad})$ & $75 / 75$ \\
$e^{+}$bunches $\varepsilon_{N r} / \varepsilon_{N \theta}(\mathrm{mm} \mathrm{rad})$ & $5 / 5$ \\
Drive $e^{-}$bunch length $(\mu \mathrm{m})$ and $N_{b}$ & 26.0 and $1.8 \times 10^{9}$ \\
Trailing $e^{-}$bunch length $(\mu \mathrm{m})$ and $N_{b}$ & 6.5 and $4.5 \times 10^{8}$ \\
Drive $e^{+}$bunch length $(\mu \mathrm{m})$ and $N_{b}$ & 26.0 and $9.0 \times 10^{7}$ \\
Trailing $e^{+}$bunch length $(\mu \mathrm{m})$ and $N_{b}$ & 6.5 and $2.3 \times 10^{7}$ \\
Transverse beam size $\left(e^{-} / e^{+}\right) \sigma_{r}(\mu \mathrm{m})$ & 10 \\
Bunch spacing $\left(e^{-} / e^{+}\right)(\mu \mathrm{m})$ & 130 \\
Preionized plasma density $\left.(\mathrm{cm})^{-3}\right)$ & $5 \times 10^{16}$ \\
Collisionless skin depth $c / \omega_{p}(\mu \mathrm{m})$ & 23.7 \\
Simulation box size $\left(c / \omega_{p}\right)$ & $20 \times 4$ \\
Cell size $\left(c / \omega_{p}\right)$ & $d z=0.05, d r=0.02$ \\
Number of particles per cell & Plasma: 4, beam: 25 \\
Time step $d t\left(1 / \omega_{p}\right)$ & 0.017 \\
\hline \hline
\end{tabular}

\section{SIMULATION OF POSITRON TRAPPING AND ACCELERATION IN THE PLASMA WAKE}

To study some outstanding physical issues related to positron trapping and acceleration in the four-bunch scheme, we performed a set of simulations exploring beam and plasma parameter space using the 2D cylindrically symmetric version of the code OSIRIS [15]. The physical parameters for the particle beams are derived from the 3D EGS5 simulation results. The energy spectrum of the positron bunch is approximately described by a halfGaussian distribution with a rms energy width of $120 \mathrm{MeV}$. For the 2D cylindrical simulations, the normalized emittances in the $r$ and $\theta$ directions are calculated from those in the $x$ and $y$ directions according to $\varepsilon_{N r}=\varepsilon_{N \theta}=$ $\sqrt{\left(\varepsilon_{N x}^{2}+\varepsilon_{N y}^{2}\right) / 2}$ assuming $\varepsilon_{N r}=\varepsilon_{N \theta}$. The default physical and numerical parameters for the simulations presented in this paper are listed in Table I.

\section{A. Trapping energy threshold}

With a plasma wave excited by a relativistic beam driver, the phase velocity of the plasma wave is equal to that of the driver and thus close to the speed of light. Therefore, the positrons need a significant initial forward energy to be trapped in the plasma wave. The EGS5 simulation results show that the number of positrons produced in the foils decreases dramatically with energy, and there are many relatively low-energy positrons. To find the energy threshold for trapping positrons in the plasma wave, we performed a set of simulations with various single energy positron bunches, keeping all other parameters the same. The trapping efficiency is defined here as the number of trapped positrons divided by the number of positrons pro- duced in the foil target. Figure 3 shows that the trapping efficiency is close to $100 \%$ for energies larger than $5 \mathrm{MeV}$.

The trapping energy threshold can also be analyzed from the 1D linear particle trapping theory [16], and expressed for the relativistic factor of the particles as

$$
\gamma \approx \gamma_{\mathrm{ph}}^{2}\left\{\varepsilon+1 / \gamma_{\mathrm{ph}}-\beta_{\mathrm{ph}}\left[\left(\varepsilon+2 / \gamma_{\mathrm{ph}}\right) \varepsilon\right]^{1 / 2}\right\}
$$

where $\gamma_{\mathrm{ph}}$ is the relativistic factor of the plasma wave and equal to that of the drive bunch, $\beta_{\mathrm{ph}}=\sqrt{1-1 / \gamma_{\mathrm{ph}}^{2}}, \varepsilon$ is the normalized plasma wave amplitude and defined as $e E_{z} / m c \omega_{p}$ ( $E_{z}$ is the plasma wake amplitude). With the parameters of Table I, $\gamma_{\mathrm{ph}}=56000, \varepsilon=0.25, \gamma=5$ and

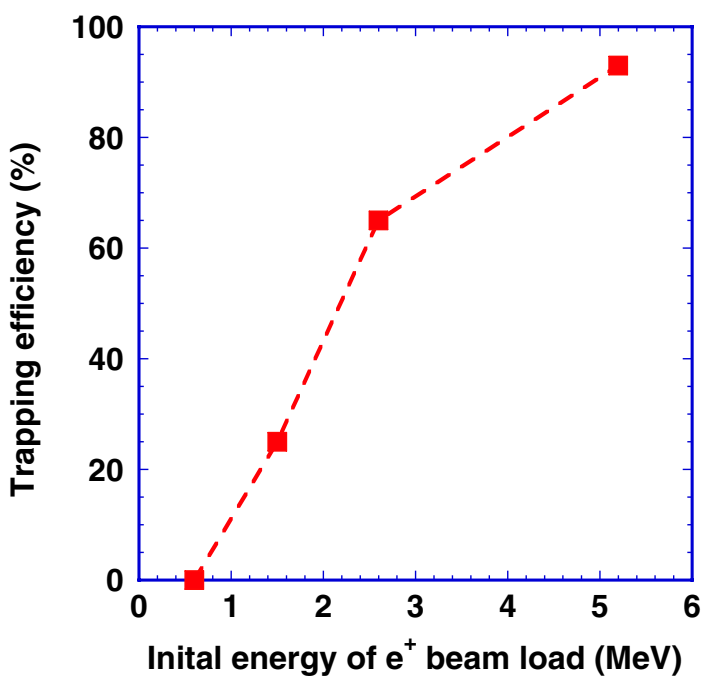

FIG. 3. (Color) Positron trapping efficiency versus the initial energy of the positron beam load energy. The plasma length is $9.5 \mathrm{~cm}$. 
corresponds to an energy of $2 \mathrm{MeV}$, which is in reasonable agreement with the simulation result seen in Fig. 3. The EGS5 simulation results [Fig. 2(a)] show that more than $99 \%$ of the positrons produced in the $0.5 \mathrm{~mm}$ tantalum target are born with forward energies larger than $5 \mathrm{MeV}$. Therefore, the loss of positrons due to their initial energy is negligible in the relativistic plasma wake.

\section{B. Influence of the nonlinearity level of the plasma wake}

In a highly nonlinear plasma wake driven by an electron beam with the beam density much larger than the plasma density [17], a wide ion column and a narrow plasma electron pinch region with a high density are created. This structure provides a large focusing phase region for electrons, but is not favorable for focusing positrons because of the small plasma electron pinch region. On the other hand, a linear plasma wake can provide a larger focusing phase region, but a smaller acceleration gradient. To find the optimal nonlinearity level of the plasma wake to focus and accelerate positrons, we perform a set of simulations in which we vary the ratio of the beam and plasma density by changing the number of electrons in the driver from $9 \times 10^{8}$ to $1.8 \times 10^{10}$ while keeping the plasma density at $5 \times 10^{16} / \mathrm{cm}^{3}$. The corresponding ratios of the peak density of the electron driver to the plasma density are varied from 0.44 to 8.8 .

Figure 4(a) shows that the number of positrons trapped into the plasma wake decreases with the number of electrons in the driver, as expected. This is because the volume region for both accelerating and focusing positrons decreases as the nonlinearity level of the plasma wake increases. We also notice that for an electron driver with a very small charge $\left(N_{b}=9 \times 10^{8}\right)$, the number of trapped positrons decreases. In this case, the plasma electron density perturbation in the pinch region is so small that the created plasma wake is not able to fully focus the trapped positrons. Figure 4(b) shows that the relative energy spread of the positron beam load increases with the number of electrons in the driver. The highly nonlinear regime $\left(N_{b}=\right.$ $\left.1.8 \times 10^{10}, n_{b} / n_{p} \gg 1\right)$ creates a large blowout radius. As a result, the positrons trapped in a radially wide plasma electron pinch region experience a large range of acceleration gradients. In the modestly nonlinear regime $\left(N_{b}=\right.$ $1.8 \times 10^{9}, n_{b} / n_{p} \approx 1$ ), a relatively narrow plasma pinch region in the radial direction provides a more uniform acceleration gradient for the trailing positrons. Therefore, the energy spread of the positron beam load in this region is smaller. For this reason, we push positron accelerator parameters into the modestly nonlinear regime, thereby increasing the number of trapped positrons and decreasing their energy spread, while sacrificing some accelerating gradient.

\section{Influence of positron beam loading}

Electron beam loading on a plasma wake has been studied theoretically and numerically in the linear [18] and nonlinear [19] regimes. However, the case of positron beam loading on the wake of an electron beam is more complicated because it involves the pinched plasma electron and the electron and positron beam loads.

First, we estimate the maximum number of positrons that can be loaded on a plasma wake according to the $1 \mathrm{D}$ linear beam loading theory [20]. Note that the linear theory applies equally to electrons and positrons in the plasma wake as it employs only the principle of conservation of energy. The engineering formula is given by

$$
N \approx 5 \times 10^{5} \frac{n_{1}}{n_{0}} \sqrt{n_{0}} A,
$$

where $n_{0}$ is unperturbed plasma density in $\mathrm{cm}^{-3}, n_{1}$ is plasma density perturbation in $\mathrm{cm}^{-3}$, and $A$ is cross section area in $\mathrm{cm}^{2}$ occupied by the beam load. In this example, the
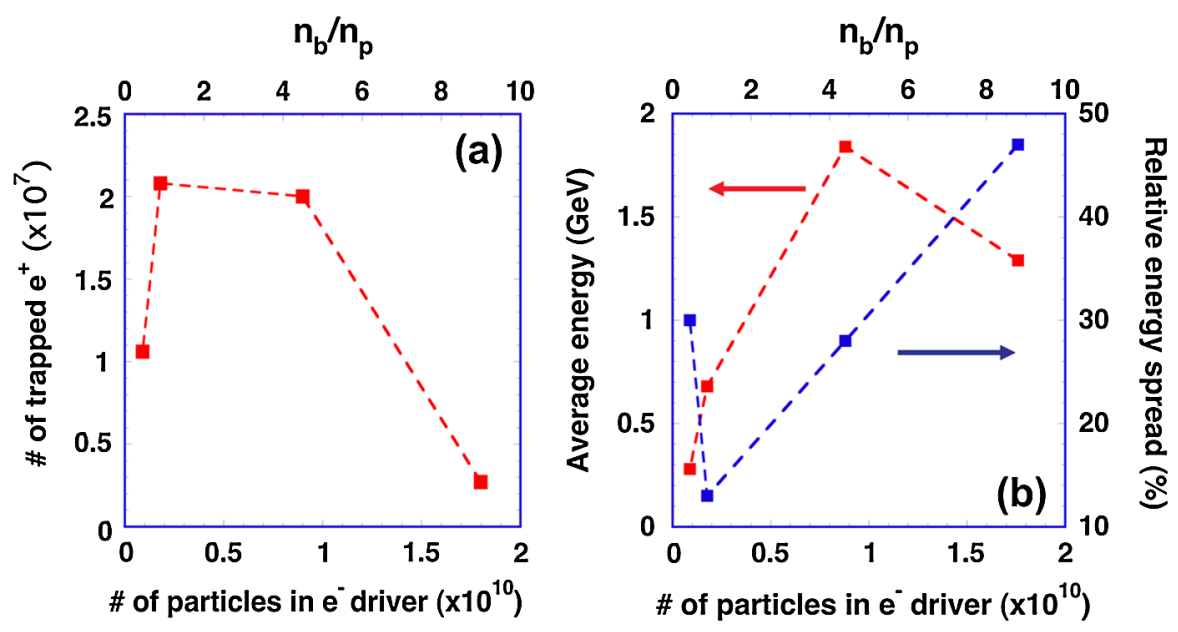

FIG. 4. (Color) (a) Number of positrons and (b) average energy (red square) and relative energy spread (blue square) of the positron beam load versus the number electrons in the driver. 
plasma density $\left(n_{0}\right)$ is $5 \times 10^{16} / \mathrm{cm}^{3}\left(n_{1} / n_{0} \sim 1\right)$, the blowout radius is $25 \mu \mathrm{m}$, and $N$ is on the order of $2 \times$ $10^{9}$. Notice that this is only an estimate since $n_{1} / n_{0} \sim 1$ invalidates the premise of the linear theory.

Next, we numerically investigate the positron beam loading effect on the plasma wake. We perform a set of simulations with a different number of particles in the trailing bunches with all other parameters fixed to their default value in Table I. The number of positrons in the beam load is varied from $2 \times 10^{7}$ to $6.8 \times 10^{8}$, which is smaller than the maximum number of positrons that can be loaded on the plasma wake according to the above 1D linear estimate. The number of electrons in the trailing bunch that generates the positrons is also simultaneously varied from $4 \times 10^{8}$ to $1.36 \times 10^{10}$, to preserve the $5 \%$ yield obtained in Sec. II. Figure 5(a) shows that while the number of trapped positrons increases with the beam load population, the trapping efficiency decreases. The trapping efficiency is about $90 \%$ for $2 \times 10^{7}$ positrons in the beam load, and drops to $30 \%$ for $6.8 \times 10^{8}$ positrons. This is due to the fact that the focusing force on the positron beam load decreases as the trailing electron bunch is defocused by the plasma wakefield. Therefore and as expected, the relative loss of positrons increases with the number of electrons in the trailing bunches, as indicated by Fig. 5(a). Figure 5(b) shows that, due to the beam loading effect, the average energy of the positron beam load decreases with the number of positrons in it. Figure 5(c) shows that the rms energy spread of the positron beam load is minimized for $2.3 \times$ $10^{8}$ positrons in the beam load. This can be explained by the accelerating field lineout along the $z$ axis [Fig. 5(d)]. Figure 5(d) show that with the optimal number of positrons (green curve) the plasma wake is locally flattened at the beam load position $(Z \approx 150 \mu \mathrm{m})$, which leads to the minimum energy spread [Fig. 5(c)]. For a larger/smaller number of positrons (red/blue curves), the positron beam over/under loads the plasma wake, which results in a larger energy spread. We note that shaping the beam load profile could possibly further reduce the energy spread.

\section{Influence of the bunch spacing}

For the typical set of parameters of Table I, the length of the positron beam load is $6.5 \mu \mathrm{m}$ and the extent of the positron trapping region of the plasma wake is about $10 \mu \mathrm{m}$ in the longitudinal direction. Therefore, we perform a set of simulations to investigate how the spacing between the drive and trailing bunch influences the number of positrons that can be trapped into the plasma wake and the quality of the accelerated beam. We vary the bunch spacing from 124 to $136 \mu \mathrm{m}$ and fix the other parameters.
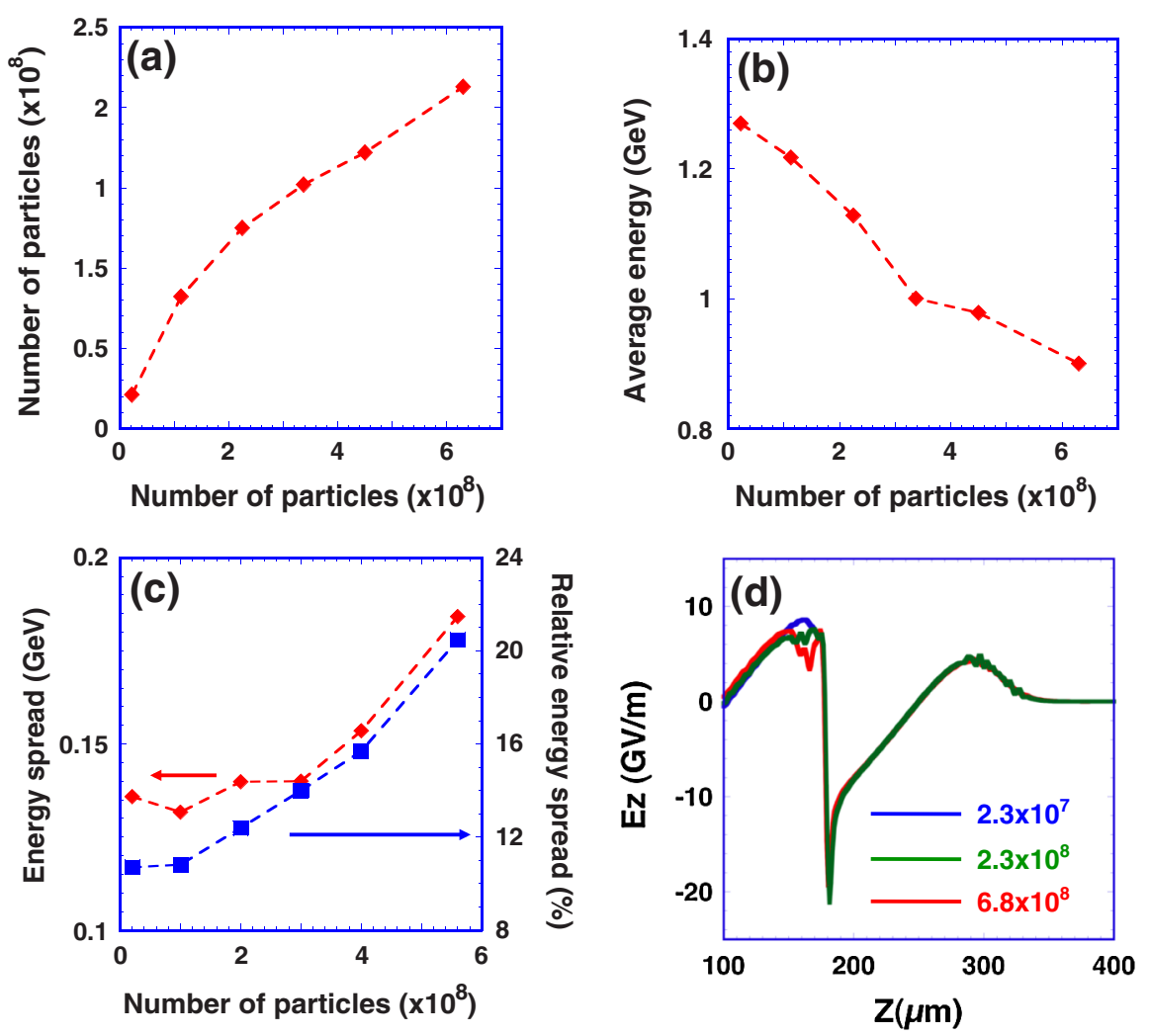

FIG. 5. (Color) (a) Number of trapped particles, (b) average energy, and (c) relative energy spread (blue squares) and energy spread (red diamonds) of the positron beam load versus the number of initial positrons in the beam load after the foil target. (d) Longitudinal wakefield lineout along the $Z$ axis after $23 \mathrm{~cm}$ propagation distance in the plasma for $2.3 \times 10^{7}$ (blue curve), $2.3 \times 10^{8}$ (green curve), and $6.8 \times 10^{8}$ (red curve) initial positrons in the beam load. 

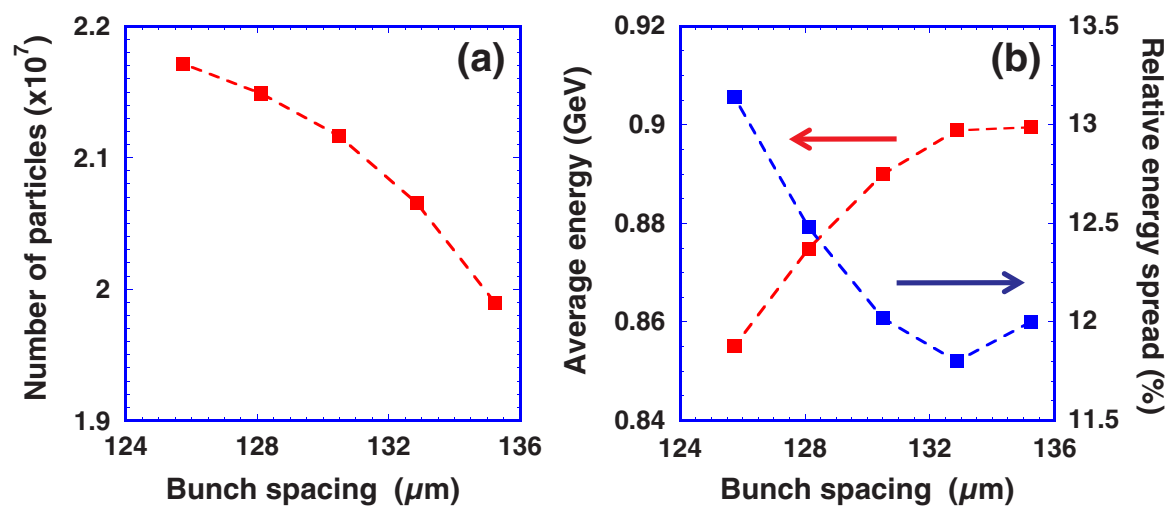

FIG. 6. (Color) (a) Number of trapped positrons and (b) average energy (red square) and relative energy spread (blue square) of the positron beam load versus the bunch spacing after $10 \mathrm{~cm}$ propagation distance in the plasma. Note the small range for the three vertical scales.

The simulation results show that number of trapped positrons [Fig. 6(a)] slightly decreases with the bunch spacing. This is due to the fact that the weakly relativistic positron beam load dephases with the highly relativistic plasma wake at the early stage of the plasma. For a small bunch spacing, the off-phase positron beam load slips backward into the trapping phase region. For a large bunch spacing, the positron beam load slips backwards into the ion column of the second blowout bubble and are lost. The simulation results also show that the average energy and relative energy spread [Fig. 6(b)] are not very sensitive to the bunch spacing in this range. This relaxes the bunch spacing requirement and makes it easier to experimentally test this acceleration scheme.

\section{E. Influence of the plasma density ramp-up}

In the particle beam driven PWFA experiments at SLAC, the self-field of the high-current electron beam ionizes a neutral $\mathrm{Li}$ vapor in a heat-pipe oven $[21,22]$. Because the pressure of the He buffer gas confines the $\mathrm{Li}$ vapor, the $\mathrm{Li}$ density increases from zero to a full density in a $10 \mathrm{~cm}$ ramp-up region at the entrance and exit of the oven. The variation of the plasma density changes the plasma wavelength and thus affects the optimal positron beam loading position. To study the dependency of the number of trapped positrons in the beam load on the length of the ramp-up region, we perform a set of simulations for a linear rampup region from zero to the full plasma density in lengths from $0 \mathrm{~cm}$ (no ramp) to $0.4 \mathrm{~cm}$. The target foil is placed at the beginning of the ramp-up where the plasma density is zero. Figure 7(a) shows that the number of trapped positrons decreases dramatically with the length of the ramp-up region, and there are less than $10^{6}$ positrons trapped for a length larger than $0.4 \mathrm{~cm}$ that is much shorter than in the actual oven $(10 \mathrm{~cm})$. In the low plasma density region the plasma wavelength is much longer than the bunch spacing, and the positron beam load is thus in the region of the ion column that strongly defocuses it. Therefore, the positrons loss increases rapidly with the length of the ramp-up region as seen in Fig. 7(a). Figure 7(b) shows that the average energy of the positron beam load decreases with the length of the ramp-up region since it is initially located in a decelerating region of the wake in the low-density plasma.
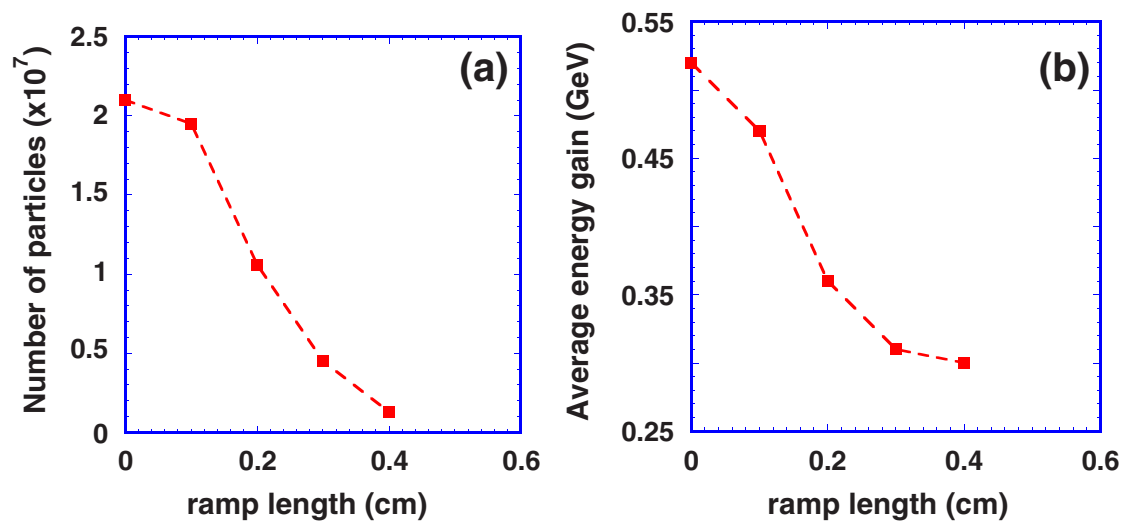

FIG. 7. (Color) (a) Number of positrons trapped and (b) their average energy versus the length of the plasma density ramp-up region after $12 \mathrm{~cm}$ of propagation distance in the plasma. 
These results clearly show the necessity of placing the foil target inside the full density plasma to maximize the positron beam load charge.

\section{F. Trapping in self-ionized plasmas}

In the previous sections, the proposed concept for positron trapping and acceleration was tested in a preionized plasma. In the recent PWFA experiments, the plasma was created by ionizing a $\mathrm{Li}$ vapor using the space charge field of a high-current drive beam [21,22]. We now explore the application of this scheme in a field-ionized plasma. The simulation parameters are the same as in Table I except the number of electrons in the drive beam is raised to $9 \times 10^{9}$ so that its space charge field becomes large enough to ionize a large volume of $\mathrm{Li}$ gas. With these parameters, the plasma wake is also in the modestly nonlinear regime [23]. This is because the amplitude of the plasma wake in the field-ionized plasma excited by a drive beam containing $9 \times 10^{9}$ electrons is about a factor of 2 less than that in the preionized plasma due to the smaller effective beam charge available to drive the plasma wake. Figure 8(a) shows that in the field-ionized plasma case, the average energy of the positron beam load increases linearly with the plasma length as in the preionized plasma case, but it saturates after $35 \mathrm{~cm}$. This saturation is because a significant level of beam head erosion pushes the ionization front backwards after a long propagation and so does the region for acceleration and focusing the positron beam load [24]. Figure 8(b) shows that the phase slippage is about $20 \mu \mathrm{m}$ between 20 and $40 \mathrm{~cm}$ of propagation. The effect of the beam head erosion in this example is much more pronounced than that in the E-167 experiments with $2 \times$ $10^{10}$ electrons in the driver [25]. With the lower bunch population, the ionization and thus the wake excitation happen later along the bunch, as the charge in the front of the bunch is not efficiently used. There are several ways to reduce the growth rate of the beam head erosion, includ- ing using lower emittance beams, applying external magnetic fields to focus the beam head in the neutral gas, and propagating in a partially preionized plasma. Beam head erosion is not a fundamental issue that limits the acceleration length as seen in the previous simulations for preionized plasmas, in which the plasma provides a focusing or a guiding force for the drive electron beam as soon as the wake excitation starts along the bunch, and is not delayed by the field ionization of the $\mathrm{Li}$ vapor.

\section{CONCLUSION}

The four-bunch scheme described here to accelerate a positron beam load on the wake of an electron beam integrates positron generation, trapping, and acceleration into a single stage. It is a promising approach for testing high-gradient acceleration of positrons in the plasma wake driven by an electron bunch before suitable electron/positron become available from conventional accelerators. It uses a proven mask technique to produce the two-bunch electron train. We have shown with $2 \mathrm{D}$ simulations that the energy spread of the positron beam load can be reduced by optimal beam loading, and that the number of trapped positrons can be maximized by using a modestly nonlinear plasma wake driven by a less intense electron beam. The optimal beam and plasma parameters for positron acceleration using this scheme are listed in Table I, and are based on parameters available at the Stanford Linear Accelerator Center. Simulations show that, with the optimal beam and plasma parameters, $\sim 10^{8}$ positrons are trapped and accelerated to $\sim 5 \mathrm{GeV}$ over $1 \mathrm{~m}$ with $\sim 5 \%$ the relative energy spread and the normalized emittances of $\sim 20 \mathrm{~mm}$ mrad in the $x$ and $\sim 25 \mathrm{~mm}$ mrad in the $y$ direction (quoted as rms momentum multiplied by rms spot size). Simulations show that some phase tolerance for positron beam loading exists in this scheme, and that placing a foil target inside the plasma is necessary to efficiently capture the low-energy positrons produced in
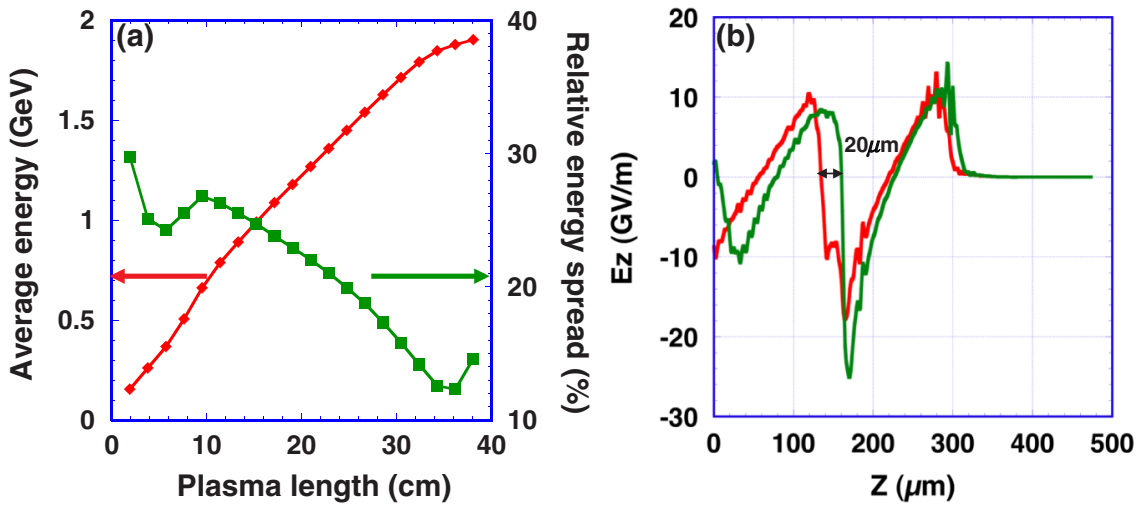

FIG. 8. (Color) 2D cylindrical simulation results in the self-ionized plasma; (a) evolution of average energy (red diamond) and relative energy spread (green square) of the positron beam load along the plasma length and (b) longitudinal wakefield $\left(E_{z}\right)$ lineout along the $Z$ axis after $20 \mathrm{~cm}$ (green line) and $40 \mathrm{~cm}$ (red line) propagation distance in the Li plasma. 
the foil target. Therefore, this scheme provides an opportunity to experimentally study positron trapping and acceleration on the plasma wake driven by an electron beam. To extend this scheme to a high-energy linear collider with a high luminosity requires increasing the number of particles and reducing the energy spread and the normalized emittance. There are several options for increasing the number of trapped positrons and the luminosity. One is to employ a high repetition rate accelerator while keeping the electron driver bunch charge relatively modest as in the above examples. Or we can lengthen the electron driver bunch length and lower the plasma density to increase the number of trapped positrons but somewhat sacrificing the acceleration gradient.

\section{ACKNOWLEDGMENTS}

This work was supported by Department of Energy Contracts No. DE-FC02-01ER41192, No. DE-AC0276 SF00515 (SLAC), No. DE-FG03- 92ER40745, No. DE-FG52-06NA26195, No. DE-FG0392ER40727, No. DE-AC-0376SF0098, No. DE-FG02-03ER54721, and National Science Foundation Grants No. ECS9632735, No. DMS-9722121, and No. PHY-0078715. Simulations were done at the USC Center for High Performance Computing and Communications (HPCC). Useful discussions with the members of the E-167 collaboration at SLAC are greatly acknowledged.
[1] S. Lee et al., Phys. Rev. ST Accel. Beams 5, 011001 (2002).

[2] I. Blumenfeld et al., Nature (London) 445, 741 (2007).

[3] P. Muggli et al., Phys. Rev. Lett. 93, 014802 (2004).

[4] M. J. Hogan et al., Phys. Rev. Lett. 95, 054802 (2005).

[5] S. Lee et al., Phys. Rev. E 64, 045501 (2001).

[6] J. S. T. Ng et al., Phys. Rev. Lett. 87, 244801 (2001).

[7] M. J. Hogan et al., Phys. Rev. Lett. 90, 205002 (2003).

[8] B. Blue et al., Phys. Rev. Lett. 90, 214801 (2003).

[9] P. Muggli et al., Phys. Rev. Lett. 101, 055001 (2008).

[10] K. V. Lotov et al., Phys. Plasmas 14, 023101 (2007).

[11] X. Wang et al., Phys. Rev. Lett. 101, 124801 (2008).

[12] P. Muggli et al., Phys. Rev. Lett. 101, 054801 (2008).

[13] M. J. Hogan et al., FACET proposal, 2008 (unpublished).

[14] H. Hirayama et al., SLAC Report No. SLAC-R-730, 2005.

[15] R. Hemker et al., Proceedings of the 1999 Particle Accelerator Conference (IEEE, Piscataway, NJ, 1999), p. 3672.

[16] T. Katsouleas and J. M. Dawson, UCLA Report No. IPFRPPG-1121, 1988.

[17] J. B. Rosenzweig et al., Phys. Rev. Lett. 58, 555 (1987).

[18] S. Wilks, T. Katsouleas et al., IEEE Trans. Plasma Sci. 15, 210 (1987).

[19] M. Tzoufras et al., Phys. Rev. Lett. 101, 145002 (2008).

[20] T. Katsouleas et al., Part. Accel. 22, 81 (1986).

[21] P. Muggli et al., IEEE Trans. Plasma Sci. 27, 791 (1999).

[22] C.L. O'Connell et al., Phys. Rev. ST Accel. Beams 9, 101301 (2006).

[23] S. Deng et al., Phys. Rev. E 68, 047401 (2003).

[24] M. Zhou, Ph.D. dissertation, UCLA, 2008.

[25] M. Zhou et al. (to be published). 